\title{
Relationship between constant strain rate and stress relaxation behavior of polypropylene
}

\author{
P. Nagy*, L. M. Vas \\ Department of Polymer Engineering, Budapest University of Technology and Economics, H-1111 Budapest, Hungary
}

Received 5 December 2006; accepted in revised form 19 January 2007

\begin{abstract}
A new method based on variable transformation is proposed for the estimation of the constant strain rate tensile test by using a previously introduced concept of viscoelastic response given to the real relaxation stimulus.

The time range of the 'good' fitting is 2.5 to 3 times larger than the best results achieved using linear viscoelastic approximations. The theoretical background of the method was elucidated as well.
\end{abstract}

Keywords: mechanical properties, viscoelasticity, stress relaxation, tensile test, polypropylene

\section{Introduction}

The mechanical response of real polymers are basically of nonlinear character, therefore their behavior patterns do not meet the idealized (linear) ones, especially in case of large loads. Nevertheless, linear viscoelasticity is often used successfully for describing the real behavior in case of small or moderate loads. The use of thermoplastics in structural applications demands accurate design data that spans appropriate ranges of stress, strain rate, time and temperature. A number of theoretical and experimental studies [1-4] have mainly dealt with the investigation of the relaxation or creep phenomena and in some cases with the relationships between the results of different tests in order to estimate or predict the short and long term behavior of polymers under different conditions. Some researchers use molecular dynamics calculations [5-7] to study the stress-strain behavior but the phenomenological or semi-phenomenological approach is more widespread [9-16]. The stress relaxation behavior of polymer materials has been studied in several theoretical and experimental investigations such as those by Urzumtsev and
Maksimov [9], Retting [10], Wortmann and Shultz [13], Andreassen [14], as well as Baeurle et al. [15]. Sudduth [2] have dealt with the relationships between creep, stress relaxation and constant strain rate data. Grzywinski and Woodford [3] predicted the creep behavior from constant stress rate tensile experiments.

In accordance with the usual loading modes the most frequently used quasi-static mechanical tests are the constant strain rate tensile, the creep, and the stress relaxation ones. Among them the tensile test lasts for the shortest time and provides an increasing load up to the damage or fracture of the specimen. During the tensile test the specimen, so to say, lives its life to the very end in an accelerated way, consequently this test may give a lot of information about the material and its mechanical behavior for various loading modes. Therefore, it may be expected that from a tensile test the behavior for the medium or long term relaxation or creep and vice versa can be predicted. In [10] Retting summarized his method and experimental results on the estimation of the real stress relaxation function as the product of the linear response and a

*Corresponding author, e-mail: nagy @pt.bme.hu

(C) BME-PT and GTE 
strain dependent factor. The tensile load curve is estimated as the time integral of the stress relaxation curve in accordance with the linear viscoelastic theory.

In this paper a new method is proposed for the estimation of the real tensile load response by using the viscoelastic response given to the real relaxation stimulus. This method can be considered as the solution of the inverse problem to estimate the (long term) stress relaxation function from the (short term) tensile test. Solving this inverse problem is mathematically somewhat less complicated and facilitates the finding of the proper functions for the direct problem. This method is completely different from the one previously published in [16] where a simple function with three adjustable parameters was used, while here a more sophisticated variable transformation method is elaborated to realize the nonlinear behavior. Variable transformations are successfully used for example in the mathematical formulation of time-temperature correspondence (the WLF Equation) [8].

\section{Experimental procedure}

Isotactic polypropylene homopolymer (Tipplen $\mathrm{H}$ $543 \mathrm{~F}$ from TVK, Hungary) having a Melt Flow Index (MFI, $2.16 \mathrm{~kg} / 230^{\circ} \mathrm{C}$ ) of $4 \mathrm{~g} / 10$ min was used for the tests. Dumbbell specimens were injection molded according to ISO 294-2 Standard on an Arburg Allrounder 320 C 600-250 injection molding machine of specimen length $148 \mathrm{~mm}$, width $10 \mathrm{~mm}$ and thickness $4 \mathrm{~mm}$. Uniaxial tensile stress relaxation tests were performed on a Zwick Z005 tensile testing machine. The tensile force was measured by a $5 \mathrm{kN}$ nominal capacity standard load cell.

Tensile tests were performed on 5 test specimens with crosshead speed of $5 \mathrm{~mm} / \mathrm{min}$ at room temperature. For the relaxation tests specimens were stretched up to $0.08,0.16,0.24,0.50,0.75$ and $1.00 \%$ engineering strains $\left(\varepsilon_{0}\right)$ with crosshead speed $(v)$ of $5 \mathrm{~mm} / \mathrm{min}$. No necking of specimens was observed in the relaxation tests. Every test was performed on a new specimen. The engineering yield strain was calculated from the crosshead displacement as $\varepsilon_{0}=\Delta l / l_{0}$ where $l_{0}$ is the gauge length at time 0 and $l_{0}=110 \mathrm{~mm}$. On the basis of these considerations the strain rate was $\dot{\varepsilon}_{0}=v / l_{0}=$ $0.0455 / \mathrm{min}=4.55 \% / \mathrm{min}$ in each case. We are aware that there are more precise methods - like strain gages or contactless CCD camera + image processing - for the strain measurements, but in this work we wanted only to demonstrate the applicability of our global approach as a predictive method and for this purpose any measured strain value is suitable.

\section{Theoretical background}

Figure 1 shows the general scheme of mechanical tests where $S$ is the specimen as a black box system, $X$ is the input stimulus and $Y$ is the response of the specimen. The two latter are time-functions.

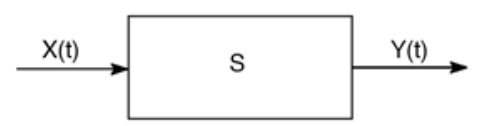

Figure 1. General scheme of mechanical tests [16]

If $S$ is an operator characterizing the material, that is the specimen, the response can be obtained as follows:

$Y(t)=S[X](t)$

In Equation (1) input $X(t)$ and output $Y(t)$ signals can be the stress and strain or vice versa, and $S$ is an operator characterizing the material. In tensile tests usually the force-time $(F(t))$ and elongation-time $(\Delta l(t))$ curves are recorded instead of stress-strain curves, so it is more natural to make the further calculations in terms of $F(t)$ and $\Delta l(t)$. The relationship between force $(F)$ and stress $(\sigma)$ and elongation $(\Delta l)$ and strain $(\varepsilon)$ is the following:

$F(t)=A_{0} \sigma(t)$

$\Delta l(t)=l_{0} \varepsilon(t)$

where $A_{0}$ and $l_{0}$ are the cross-sectional area and gauge length of the unloaded specimen, respectively.

Figure 2 shows the measured and estimated forcetime curves, the relations between them and the used notations for a constant strain-rate tensile experiment as well as for a stress relaxation experiment in which a real stimulus (namely, constant strain-rate) is applied. From Equation (1) it follows for the latter:

$F_{1}(t)=S\left[\Delta l_{1}\right](t)$ 


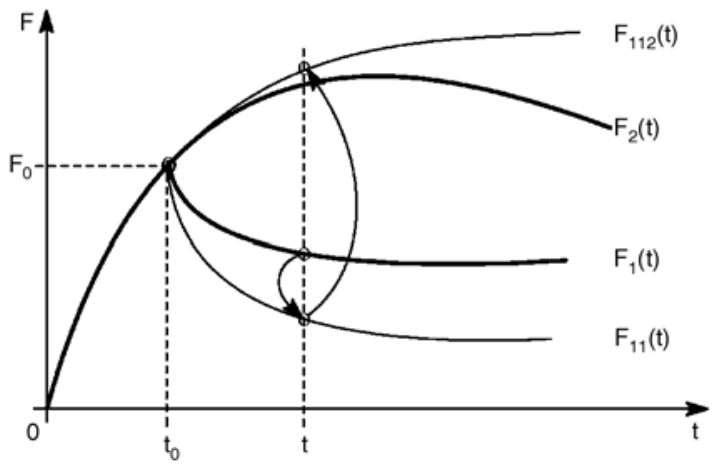

Figure 2. Measured and estimated force-time curves. $F_{2}(t)$ : measured force in a tensile test experiment; $F_{1}(t)$ : measured force in a stress relaxation experiment; $F_{11}(t)$ : force estimated from $F_{1}(t)$ in such way that it approximates correctly the derivative of the stress-strain curve $\left(F_{2}(t)\right)$ in a possibly wide range; $F_{112}(t)$ : force estimated from $F_{11}(t) ; F_{0}$ and $t_{0}$ : force and time, respectively, at the beginning of the stress relaxation experiment

Similar expressions represent the relationship between $F_{1}(t)$ and $F_{11}(t), F_{112}(t)$ and $F_{11}(t)$ and $F_{2}(t)$ and $\Delta l_{2}(t)$ :

$F_{11}(t)=S_{1}\left[F_{1}\right](t) \rightarrow$

$F_{112}(t)=S_{2}\left[F_{11}\right](t) \approx F_{2}(t)=S\left[\Delta l_{2}\right](t)$

where $S_{1}$ is a nonlinear estimating operator and $S_{2}$ is a linear estimating operator (see [16] for details). The linear mapping operator $S_{2}$ can be represented by the following recursive equation:

$F_{112}(t)=F_{112}\left(t-t_{0}\right)+F_{11}(t)$

The unknown operator $S_{1}: F_{1} \rightarrow F_{11}$ is to be chosen so that estimation $F_{112}(t)$ provides an acceptable accuracy in a given time range. It is obvious that $F_{1}$ and $F_{11}$ are similar to each other regarding the fact that they both must have the properties of a stress relaxation function (Figure 2). First of all, the initial values are identical:

$F_{11}\left(t_{0}\right)=F_{1}\left(t_{0}\right)=F_{0}$

They must be monotonously decreasing that is:

$\frac{\mathrm{d} F_{1}}{\mathrm{~d} t}<0, \frac{\mathrm{d} F_{11}}{\mathrm{~d} t}<0, t>t_{0}$

and specially:

$\dot{F}_{0}=\frac{\mathrm{d} F_{11}}{\mathrm{~d} t}\left(+t_{0}\right)<\dot{F}_{10}=\frac{\mathrm{d} F_{1}}{\mathrm{~d} t}\left(+t_{0}\right)<0$
At the same time they must be convex from below:

$$
\frac{\mathrm{d}^{2} F_{11}}{\mathrm{~d} t^{2}}>0, \frac{\mathrm{d}^{2} F_{1}}{\mathrm{~d} t^{2}}>0, t>t_{0}
$$

as well as finally they - in normal cases - have to tend to a nonnegative finite value $\left(F_{1 \infty}, F_{\infty}\right)$ :

$F_{1}(t) \rightarrow F_{1 \infty} \geq 0, t \rightarrow \infty$

$F_{11}(t) \rightarrow F_{\infty} \geq 0, t \rightarrow \infty$

where

$0 \leq F_{\infty}<F_{1 \infty}<F_{0}$

On the basis of Equation (10) $F_{1}$ and $F_{11}$ are twice continuously differentiable the set of which is denoted by $C_{2}=C_{2}\left(t_{0}, \infty\right)$. Let us denote the set of the functions meeting the condition formulated above - except for Equation (9) - by $C_{2}{ }^{*}=$ $C_{2}{ }^{*}\left(t_{0}, \infty\right) \subset C_{2}$. In this case operator $S_{1}$ can be given as a mapping as follows:

$S_{1}: C_{2}{ }^{*}\left(t_{0}, \infty\right) \rightarrow C_{2}{ }^{*}\left(t_{0}, \infty\right)$

and it can be represented for example by an integral using Equation (5) and (7):

$$
F_{11}(t)=S_{1}\left[F_{1}\right](t)=F_{0}-\int_{t_{0}}^{t} G\left(t, u, f_{1}(u)\right) \mathrm{d} f_{1}(u)
$$

where $G$ is a core-function characterizing the nonlinearity of the material and

$f_{1}(t)=F_{1}\left(t_{0}\right)-F_{1}(t)=F_{0}-F_{1}(t)$

In a special case applied in this paper as an example, if $G$ is explicitly independent of variables $t$ and $u$ operator $S_{11}$ can be simply represented by a function $(g)$ in the following form:

$F_{11}(t)=F_{1}(t)-g\left(f_{1}(t)\right)$

$g\left(f_{1}\right)=\int_{0}^{f_{1}} G(x) \mathrm{d} x$

which must meet the requirements Equation (7)(12) from which the following conditions for function $g\left(f_{1}\right) \geq 0$ can be derived for $t \geq t_{0}$ :

$F_{11}\left(t_{0}\right)=F_{11}\left(t_{0}\right)-g\left(f_{1}\left(t_{0}\right)\right)=F_{1}\left(t_{0}\right)=F_{0} \Rightarrow$ $g(0)=0$ 


$$
\begin{aligned}
& \frac{\mathrm{d} F_{11}}{\mathrm{~d} t}=-\frac{\mathrm{d} g}{\mathrm{~d} f_{1}}\left(-\frac{\mathrm{d} F_{1}}{\mathrm{~d} t}\right)<0 \Rightarrow \frac{\mathrm{d} g}{\mathrm{~d} f_{1}}>0 \\
& \dot{F}_{0}=\frac{\mathrm{d} F_{11}}{\mathrm{~d} t}\left(+t_{0}\right)=\left.\left.\frac{\mathrm{d} g}{\mathrm{~d} f_{1}}\right|_{+t_{0}} \frac{\mathrm{d} F_{1}}{\mathrm{~d} t}\right|_{+t_{0}}<\dot{F}_{10}<0 \\
& \left.\Rightarrow \frac{\mathrm{d} g}{\mathrm{~d} f_{1}}\right|_{+t_{0}}>1 \\
& \frac{\mathrm{d}^{2} F_{11}}{\mathrm{~d} t^{2}}=\frac{\mathrm{d}^{2} g}{\mathrm{~d} f_{1}^{2}}\left(\frac{\mathrm{d} F_{1}}{\mathrm{~d} t}\right)^{2}+\frac{\mathrm{d} g}{\mathrm{~d} f_{1}} \frac{\mathrm{d}^{2} F_{1}}{\mathrm{~d} t^{2}}>0 \Rightarrow \\
& -\frac{\mathrm{d}^{2} g}{\mathrm{~d} f_{1}^{2}}<\frac{\mathrm{d} g}{\mathrm{~d} f_{1}} \frac{\frac{\mathrm{d}^{2} F_{1}}{\mathrm{~d} t^{2}}}{\left(\frac{\mathrm{d} F_{1}}{\mathrm{~d} t}\right)^{2}}
\end{aligned}
$$

$F_{11}(t)=F_{0}-g\left(F_{0}-F_{1}(t)\right) \rightarrow F_{0}-g\left(F_{0}-F_{1 \infty}\right)=$

$F_{\infty} \geq 0, t \rightarrow \infty \Rightarrow$

$0<F_{0}-F_{1 \infty}<g\left(F_{0}-F_{1 \infty}\right)=F_{0}-F_{\infty}<F_{0}$

It is notable that inequality (20) is trivially fulfilled if

$\frac{\mathrm{d}^{2} g}{\mathrm{~d} f_{1}^{2}}>0$

In [16] a special $g(t)$ function was used. Another possibility of describing operator $S_{1}$ is the application of variable transformations for example in the following form:

$F_{11}(t)=S_{1}\left[F_{1}\right](t)=$

$F_{0}-b\left[F_{0}-F_{1}\left(t_{0}+\left(t-t_{0}\right) a(t)\right)\right]$

where - to a certain degree similarly to the WLF Equation $[5,6]-a(t) \geq 0$ is a kind of delay-function and $b$ is a constant. Introducing the following notation:

$h(t)=t_{0}-\left(t-t_{0}\right) a(t)$

let us suppose that

$h(t) \rightarrow \infty \Rightarrow, t \rightarrow \infty$

On the basis of Equation (11) and (25) the value of $b$ can be obtained easily:

$b=\frac{F_{0}-F_{\infty}}{F_{0}-F_{1 \infty}}>1$

and applying Equation (8)-(10) provides some other information about function $a(t)$ :

$$
\begin{aligned}
& \frac{\mathrm{d} F_{11}}{\mathrm{~d} t}=-b\left(-\frac{\mathrm{d} F_{1}}{\mathrm{~d} h}\right) \frac{\mathrm{d} h}{\mathrm{~d} t}<0 \Rightarrow \\
& \frac{\mathrm{d} h}{\mathrm{~d} t}=a(t)+\left(t-t_{0}\right) \frac{\mathrm{d} a}{\mathrm{~d} t}>0 \Rightarrow \\
& \frac{\mathrm{d} a}{\mathrm{~d} t}>-\frac{a(t)}{t-t_{0}} \\
& \frac{\mathrm{d} F_{11}}{\mathrm{~d} t}\left(+t_{0}\right)<\frac{\mathrm{d} F_{1}}{\mathrm{~d} t}\left(+t_{0}\right)<0 \Rightarrow \\
& \left.\frac{\mathrm{d} h}{\mathrm{~d} t}\right|_{+t_{0}}>\frac{1}{b} \Rightarrow a\left(t_{0}\right)>\frac{1}{b} \\
& \frac{\mathrm{d}^{2} F_{11}}{\mathrm{~d} t^{2}}=b \frac{\mathrm{d}^{2} F_{1}}{\mathrm{~d} h^{2}}\left[\frac{\mathrm{d} h}{\mathrm{~d} t}\right]^{2}+b \frac{\mathrm{d} F_{1}}{\mathrm{~d} t} \frac{\mathrm{d}^{2} h}{\mathrm{~d} t^{2}}>0 \Rightarrow \\
& \frac{\mathrm{d}^{2} h}{\mathrm{~d} t^{2}}>-\left[\frac{\mathrm{d} h}{\mathrm{~d} t}\right]^{2} \frac{\frac{\mathrm{d}^{2} F_{1}}{\mathrm{~d} h^{2}}}{\frac{\mathrm{d} F_{1}}{\mathrm{~d} h}}>0
\end{aligned}
$$

In case of linear viscoelasticity $S_{1}$ is the identical operator hence $g\left(f_{1}\right) \equiv f_{1}$ in Equation (16) and $a=1$, $b=1$ in Equation (23).

It is obvious that a stress relaxation response function of a Standard-Solid model $\left(F_{S S}\right)$ [5] fulfils conditions for $F_{1}$ or $F_{11}$ therefore $F_{S S} \in C_{2}{ }^{*}$ that is why it can be well applied as a simple test function for both $F_{1}$ and $F_{11}\left(t \geq t_{0}\right)$ :

$$
\begin{aligned}
& F_{1}(t)=F_{1 \infty}+\left(F_{0}-F_{1 \infty}\right) \mathrm{e}^{-\frac{t-t_{0}}{\tau_{1}}} \\
& F_{11}(t)=F_{\infty}+\left(F_{0}-F_{\infty}\right) \mathrm{e}^{-\frac{t-t_{0}}{\tau_{2}}}
\end{aligned}
$$

where in accordance with Equation (9) $\tau_{2}<\tau_{1}$.

The mapping ( $g$ ) according to Equation (16) can be calculated easily by expressing $t$ from $F_{1}(t)$ and substituting that into $F_{11}(t)$ :

$F_{11}(t)=F_{\infty}+\left(F_{0}-F_{\infty}\right)\left[\frac{F_{1}(t)-F_{1 \infty}}{F_{0}-F_{1 \infty}}\right]^{\frac{\tau_{1}}{\tau_{2}}}=$

$F_{0}-\left(F_{0}-F_{\infty}\right)\left(1-\left[1-\frac{F_{0}-F_{1}(t)}{F_{0}-F_{1 \infty}}\right]^{\frac{\tau_{1}}{\tau_{2}}}\right)$

from which function $\mathrm{g}$ can be determined:

$g\left(f_{1}\right)=\left(F_{0}-F_{\infty}\right)\left(1-\left[1-\frac{f_{1}(t)}{F_{0}-F_{1 \infty}}\right]^{\frac{\tau_{1}}{\tau_{2}}}\right)$ 
According to Equation (33) function $g$ is nonlinear if $\tau_{2} \neq \tau_{1}$.

The mapping according to Equation (23) can be tested similarly. If $a=\tau_{1} / \tau_{2}$ then Equation (23) produces the mapping between the $F_{1}$ and $F_{11}$ of exponential form (30):

$$
\begin{aligned}
& F_{11}(t)=S_{1}\left[F_{1}\right](t)= \\
& F_{0}-\frac{F_{0}-F_{\infty}}{F_{0}-F_{1 \infty}}\left[F_{0}-F_{1}\left(t_{0}-\left(t-t_{0}\right) \frac{\tau_{1}}{\tau_{2}}\right)\right]
\end{aligned}
$$

In case of simple exponential relaxation functions mapping ' $g$ ' according to Equation (32) much more complicated than that of function ' $a$ ' which is a constant.

It is well known that the real relaxation functions can be well approximated the response of a generalized Standard Solid model [5] which is a sum of exponential functions such as according to Equation (30):

$$
\begin{aligned}
& F_{1}(t)=F_{1 \infty}+\left(F_{0}-F_{1 \infty}\right) \sum_{i=1}^{n} \alpha_{i} \mathrm{e}^{-\frac{t-t_{0}}{\tau_{1 i}}} \\
& F_{11}(t)=F_{\infty}+\left(F_{0}-F_{\infty}\right) \sum_{i=1}^{n} \alpha_{i} \mathrm{e}^{-\frac{t-t_{0}}{\tau_{2 i}}}
\end{aligned}
$$

where $n$ is the number of the Maxwell branches in the model and $\alpha_{i}(i=1, \ldots, n)$ are weighting factors:

$$
\sum_{i=1}^{n} \alpha_{i}=1
$$

It is easy to see that Equation (33) realizes a correct mapping between functions according to Equation (34) as well if $a=\tau_{1} / \tau_{2}$ by transforming the parameters as follows:

$F_{1 \infty} \rightarrow F_{\infty}$

$\tau_{1 i} \rightarrow \tau_{2 i}=\tau_{1 i} / a$

$\alpha_{i} \rightarrow \alpha_{i}$

Unfortunately, this is not true for mapping Equation (31) because instead of the form according to Equation (34) it gives another form such as (using ' $a$ ' instead of $\tau_{1} / \tau_{2}$ ):

$$
F_{11}(t)=F_{\infty}+\left(F_{0}-F_{\infty}\right)\left[\sum_{i=1}^{n} \alpha_{i} \mathrm{e}^{-\frac{t-t_{0}}{\tau_{1 i}}}\right]^{a}
$$

On the basis of all these considerations and the fact that the real relaxation curves can be well approximated by the functions of type according to Equation (34) it may be supposed that mapping Equation (33) can be used for transforming the results of relaxation measurements as well.

\section{Fitting of the observations}

For the estimation of $F_{2}(t)$, the force-time curve of a tensile test, we start from $F_{1}(t)$, the measured force-time curve of the stress relaxation experiment (see Figure 2). First we perform a transformation of time, $t$ :

$t^{\prime}=h(t)$

where $h(t)$ is a function to be determined. This function should make the following mapping of the time axis:

$h:\left[0, t_{s}\right) \mapsto[0, \infty)$

where $t_{s}$ is the time corresponding to the yield or ultimate elongation in the tensile test. Figure 3 shows the general appearance of $h(t)$.

In the next step we calculate from $F_{1}(t)$ a transformed force-time curve, $F_{1}\left(t^{\prime}\right)=F_{1}(h(t))$.

$F_{11}(t)$ can be calculated according to Equation (23) in the following manner:

$F_{11}(t)=F_{0}-b\left[F_{0}-F_{1}(h(t))\right]$

In the last step $F_{112}(t)$, the estimate of the tensile load-time curve $\left(F_{2}(t)\right)$ is calculated from $F_{11}(t)$ according to the recursive formula of Equation (6). The parameters of the mapping function $h(t)$ as well as $b$ are estimated by fitting $F_{112}(t)$ to $F_{2}(t)$. Because the resulting equations are usually non-lin-

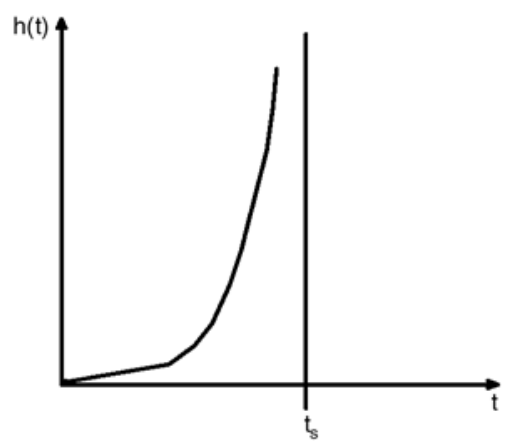

Figure 3. General appearance of $h(t)$ 
ear for the parameters, numerical procedures should be used.

We have tested the performance of our model by some simple functions that satisfy Equation (39):

$h 1(t)=t_{0}+a\left(t_{s}-t_{0}\right)\left[\frac{t-t_{0}}{t_{s}-t}\right]^{c}$

$h 2(t)=t_{0}+a\left(t-t_{0}\right)^{k} \exp \left(\frac{c}{t_{s}-t}\right)$

$h 3(t)=t_{0}+a \frac{\left(t_{s}-t_{0}\right)\left(t-t_{0}\right)}{t_{s}-t}\left(1+\frac{t_{s}-t_{0}}{t_{1}-t_{0}}\right)^{c}$

$h 4(t)=t_{0}+\left[\tan \left(\frac{t-t_{0}}{t_{s}-t_{0}}\right)\right]^{c}$

where $t_{0}$ is the time corresponding to the beginning of the stress relaxation experiment (Figure 2), $t_{s}$ is the time corresponding to the yield or ultimate elongation in the tensile test, and $a, c, k$, and $t_{1}$ are adjustable parameters. Figure 4 to 7 summarizes the results for $h 1(t)$ to $h 4(t)$.
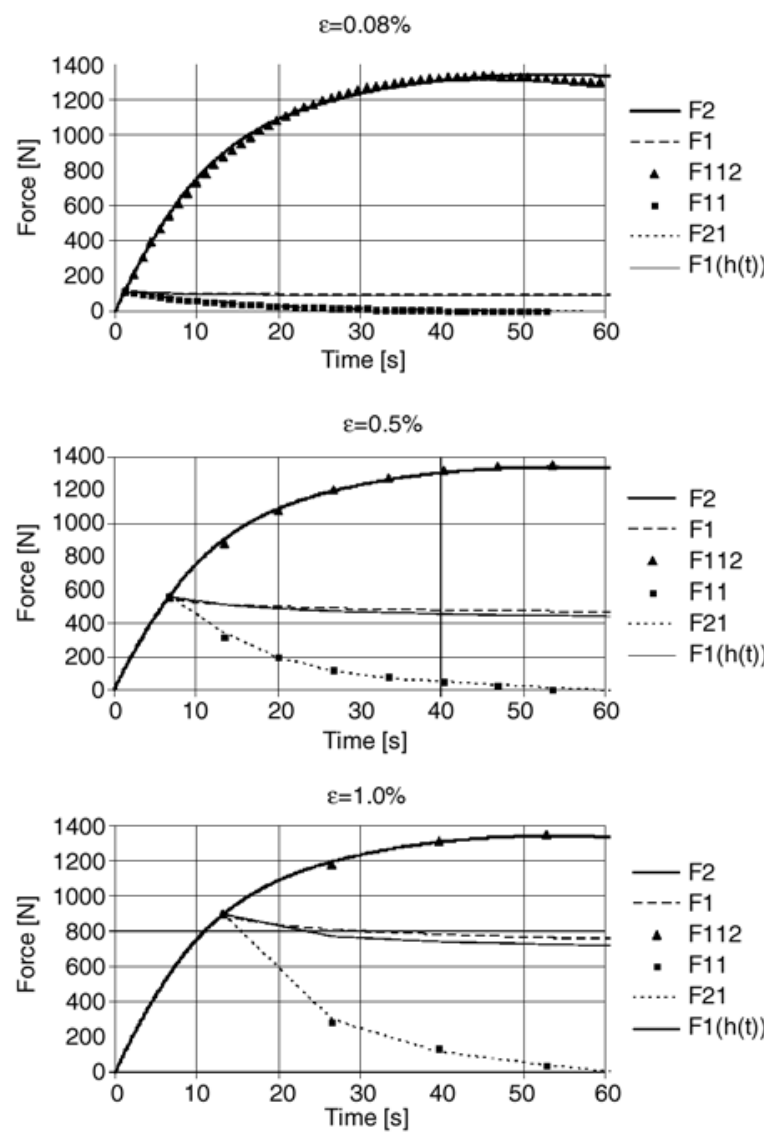

Figure 4. Measured and estimated force-time curves for 3 different initial strains. $h(t)=h 1(t)$
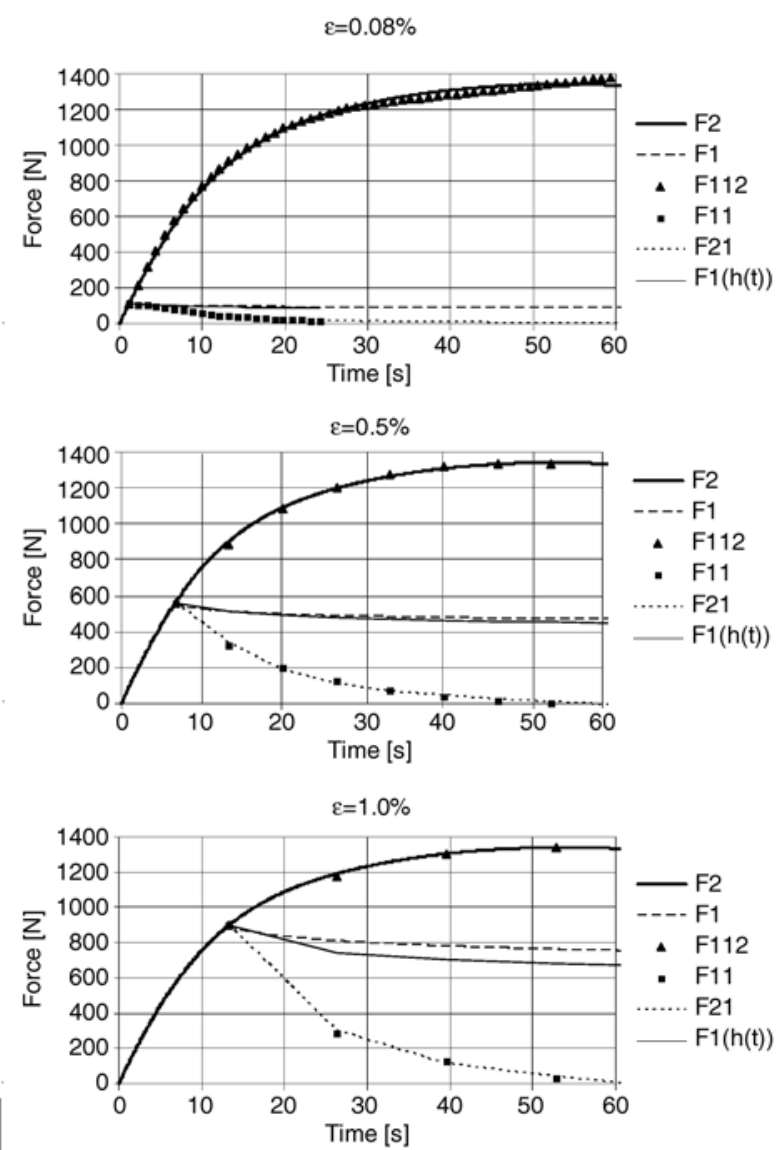

Figure 5. Measured and estimated force-time curves for 3 different initial strains. $h(t)=h 2(t)$

\section{Discussion}

As it can be seen from Figure 4-7 there is a fair agreement between the force-time curve $\left(F_{2}\right)$ measured in constant strain rate tensile test and its nonlinear viscoelastic approximation, $F_{112}$, in a relatively wide time and initial strain $(\varepsilon)$ range, concerning the duration of a tensile test. The time range (between 50 and $60 \mathrm{~s}$ for the $h(t)$-s tested) of the 'good' fitting is 2.5 to 3 times larger than the best results found for linear viscoelastic approximations (20-22 s, see [16]). Table 1 summarizes the goodness of approximation calculated in the time range tested as

$$
R^{2}=1-\frac{\sum\left(y_{i}-\hat{y}_{i}\right)^{2}}{\sum\left(y_{i}-\bar{y}_{i}\right)^{2}}
$$

where $y_{i}$ is the $i$-th measured value, $\hat{y}$ is its approximation and is the mean of the measured values. The best results for all initial strain $(\varepsilon)$ ranges were achieved by $h 2(t)$, an exponential function. 

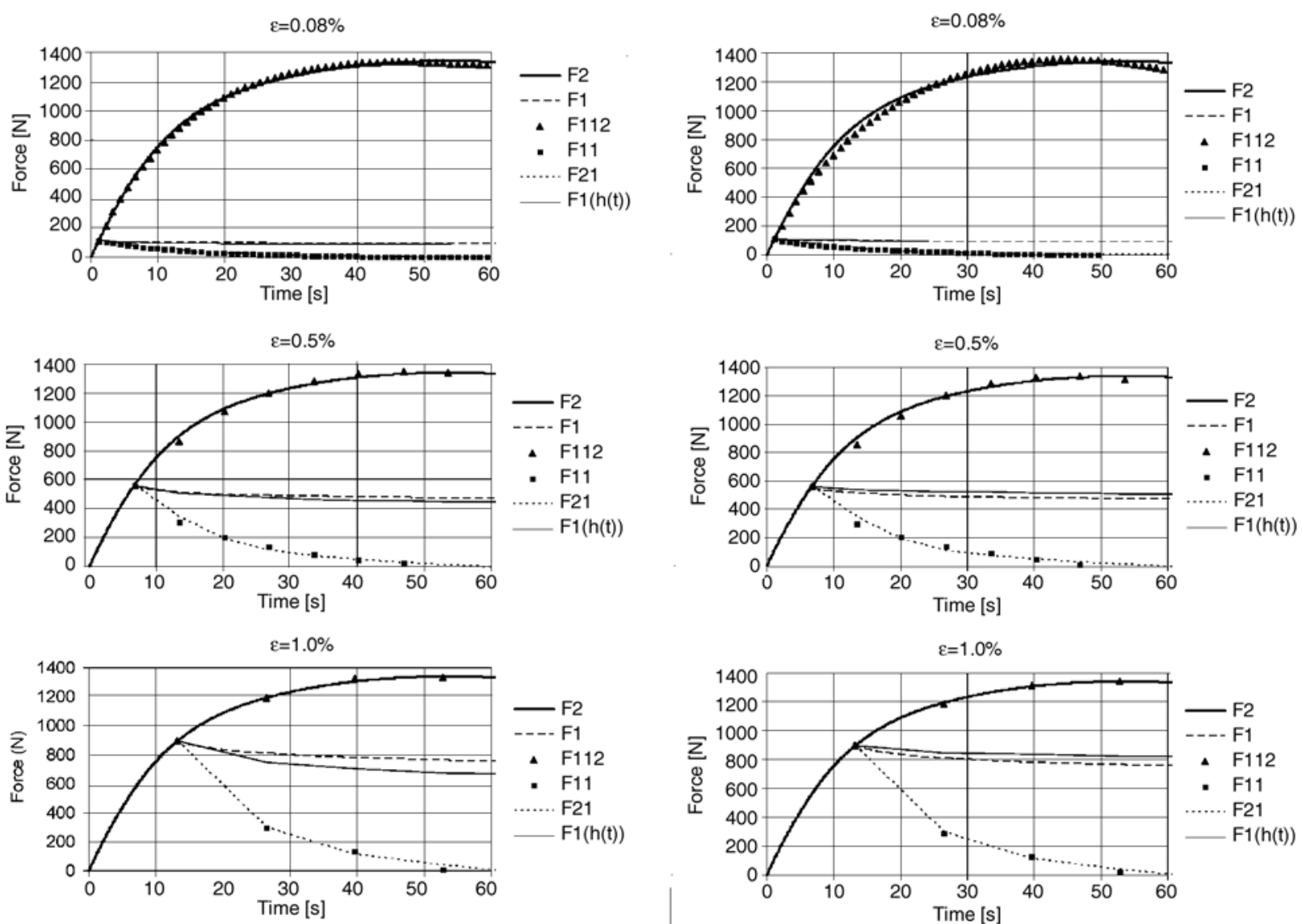

Figure 6. Measured and estimated force-time curves for 3 different initial strains. $h(t)=h 3(t)$

Table 1. Goodness of fit $\left(R^{2}\right)$ values for the four $\mathrm{h}(\mathrm{t})$ functions

\begin{tabular}{|c|c|c|c|c|}
\hline Initial strain $(\boldsymbol{\varepsilon})[\%]$ & $\mathbf{h 1}(\mathbf{t})$ & $\mathbf{h 2 ( t )}$ & $\mathbf{h 3 ( t )}$ & $\mathbf{h 4 ( t )}$ \\
\hline 0.08 & 0.9984 & 0.9994 & 0.992 & 0.9932 \\
\hline 0.5 & 0.9676 & 0.9952 & 0.9693 & 0.9280 \\
\hline 1.0 & 0.9892 & 0.9739 & 0.9627 & 0.9919 \\
\hline
\end{tabular}

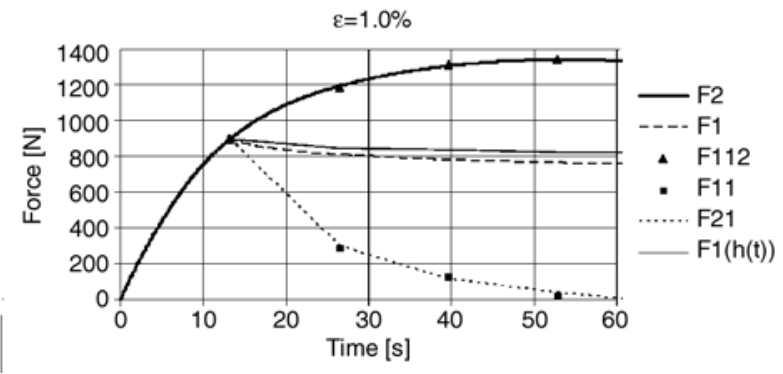

Figure 7. Measured and estimated force-time curves for 3 different initial strains. $h(t)=h 4(t)$

\section{Acknowledgements}

The authors would like to thank the OTKA Contract No. T049069 for the support of this research.

\section{References}

\section{Conclusions}

We have shown that the force-time curve of the constant strain rate tensile test can be modeled using the results of the stress relaxation experiment for a semicrystalline polypropylene material. Good results were achieved by using variable transformation as the mapping operation, especially in the case of an exponential function, $h 2(t)$. In our next paper we will show how the inverse problem modeling of the stress relaxation function from the measured constant strain rate tensile test - can be solved.

[1] Sudduth R. D.: Development of a simplified relationship between uniaxial creep, stress relaxation, and constant strain-rate results for viscoelastic polymeric materials. Journal of Applied Polymer Science, 82, 527-540 (2001).

[2] Sudduth R. D.: Comparison of the viscous and elastic components of two ABS materials with creep, stress relaxation and constant strain rate measurements using the universal viscoelastic model. Journal of Applied Polymer Science, 90, 1298-1318 (2003).

[3] Grzywinski G. G., Woodford D. A.: Creep analysis of thermoplastics using stress relaxation data. Polymer Engineering and Science, 35, 1931-1937 (1995).

[4] Karger-Kocsis J., Czigány T., Moskala E. J.: Deformation rate dependence of the essential and nonessential work of fracture parameters in an amorphous copolyester. Polymer, 39, 3939-3944 (1998). 
[5] Baeurle S. A., Usami T., Gusev A. A.: A new multiscale approach for the prediction of mechanical properties of polymer-based nanomaterials. Polymer, 47, 8604-8617 (2006).

[6] Aoyagi T., Honda T., Doi M.: Microstructural study of mechanical properties of the ABA triblock copolymer using self-consistent field and molecular dynamics. Journal of Chemical Physics, 117, 8153-8161 (2002).

[7] Terzis A. F., Theodorou D. N., Stroeks A.: Entanglement network of the polypropylene/polyamide interface. 3. Deformation to fracture. Macromolecules, 35, 508- 521 (2002).

[8] Ferry J. D.: Viscoelastic properties of polymers. Wiley and Sons, New York (1980).

[9] Urzumtsev Yu. S., Maksimov R. D.: Deformation of Plastics, Prognostics on the Basis of Similarity Principles (in Hungarian). Múszaki Könyvkiadó, Budapest (1982).

[10] Retting W.: Mechanik der Kunststoffe. Hanser Verlag, München (1992).
[11] Drozdov A. D., Agarwal S., Gupta R. K.: Linear thermo-viscoelasticity of isotactic polypropylene. Computational Materials Science, 29, 195-213 (2004).

[12] Drozdov A. D., Gupta R. K.: Non-linear viscoelasticity and viscoplasticity of isotactic polypropylene. International Journal of Engineering Science, 41, 2335-2361 (2003)

[13] Wortmann F. J., Shultz K. V.: Stress relaxation and time/temperature superposition of polypropylene fibres. Polymer, 36, 315-321 (1995).

[14] Andreassen E.: Stress relaxation of polypropylene fibres with various morphologies. Polymer, 40, 3909 3918 (1999).

[15] Baeurle S. A., Hotta A., Gusev A. A.: A new semiphenomenological approach to predict the stress relaxation behavior of thermoplastic elastomers. Polymer, 46, 4344-4354 (2005).

[16] Vas L. M., Nagy P.: Investigating the time dependent behavior of thermoplastic polymers under tensile load. Macromolecular Symposia, 239, 176-181 (2006). 